\title{
SIN ESTÁNDAR, PERO CON PRINCIPIOS: EDUCAR EN LA DIVERSIDAD
}

\author{
WITHOUT STANDARDS, BUT \\ WITH PRINCIPLES: EDUCATING IN \\ DIVERSITY
}

Francisco Coriñaupa Vargas

fcorinaupa@gmail.com

\begin{abstract}
RESUMEN
Desde muy pequeños los niños son introducidos en el mundo educativo, ya sea por los motivos que fueren, el estudiante antes de los veinte años pasó la mayor parte de su vida en un ambiente construido de forma intencional para el aprendizaje.

De lo anterior se deduce que la enorme influencia que tiene la escuela (institución, currículo, compañeros, maestros e incluso otros padres) en el estudiante y su forma de percibir el mundo, ya sea como un mundo exclusivo, al cual tiene que ingresar, o como un mundo que respeta lo diverso. En ese sentido, el éxito ya no es banal si no responde a lo que la sociedad pide como verdad, sino que es una posibilidad para construir desde su peculiaridad, respetando el principio de ser único en todo sentido, de allí que no sea posible estandarizar cómo se aprende. Educar en la diversidad, es un acto ético.
\end{abstract}

\section{PALABRAS CLAVE}

Educación en la diversidad, escucha, estándar, inclusión, diferencia, éxito.

\section{ABSTRACT}

From a very young age children are introduced to the world of education, whether for whatever reason, the student before the age of twenty spent most of his or her life in an intentionally built environment for learning.

From the above, we can deduce that the enormous influence that the school (institution, curriculum, peers, teachers and even other parents) has on the student and his or her way of perceiving the world, whether as an exclusive world, which he or she has to enter, or as a world that respects diversity. In this sense, success is no longer banal if it does not respond to what society asks for as truth, but is a possibility to build from its peculiarity, respecting the principle of being unique in every sense, hence it is not possible to standardize how one learns. Educating in diversity is an ethical act.

\section{KEYWORDS}

Diversity education, listening, standard, inclusion, difference, success.
La escuela les saca el erotismo a las palabras. Las hace simétricas, aplanadas, llanas, yermas, estériles. Aplaca aquello que atañe a la pregunta primordial sobre la condición humana... el lenguaje.

Duschatzky, S. (2017).

La política de la escucha en la escuela.

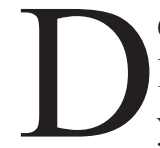
esde muy pequeños uno de los primeros lugares en ser visitados por los niños, y donde pasarán buena parte de su vida es la escuela, en ocasiones, ingresan en instituciones educativas antes que hayan dejado el pañal, y muchas veces, incluso cuando recién han empezado a caminar en la vida; es decir, cuando el estudiante llega a los veinte años, ha transcurrido la mayor parte de su vida en una institución educativa, ya sea llamada cuna, jardín, kínder, colegio, universidad o instituto $\mathrm{y}$, además, ha pasado por distintos niveles educativos -inicial, primaria y secundaria-, sin contar los estudios superiores, si es que los siguió.

En esos años, el sujeto aprendió cosas básicas de la vida y del mundo que lo rodea, ir al baño, a hablar fluidamente, aprendió de la amistad, del amor, de las decepciones, de valores como: solidaridad, honestidad, laboriosidad, entre otros; aprendió también de la tristeza, del dolor físico y del emocional, aprendió a ser empático, proactivo, crítico, aprendió a ser autónomo y trabajar de forma colaborativa, entre muchos aspectos aprendió aquello que caracteriza al 
ser humano: socializar, aprendió también que esa socialización implica muchos aspectos, conceptos e ideas propias de la cultura y de la familia, resultando la institución educativa un eje central en este proceso.

No obstante, no todos aprendimos de igual manera, y es que algunos niños y jóvenes saben lo que deben hacer, pero no lo practican, no porque no quieran, sino porque no pueden, por lo menos es lo que alguno de ellos afirma: ¿qué pasó con ellos?

De forma tradicional estamos acostumbrados a creer que la escuela es sólo un centro al cual se envían a estudiantes para que sean instruidos y/o adoctrinados con saberes, siendo esto tan importante que hay padres de familia que reclaman por 0,3 de punto o por no tener claro el puesto en que se encuentran sus hijos, y claro, esta fue otra fórmula que aprendió el niño, competir y reclamar, porque es injusto, porque pareciera que alguien le impide ser exitoso, como dicen los medios de comunicación, y como, muchas veces, creen sus padres, siendo el niño o joven solo un objeto, objeto de deseo de los padres o de la impronta social, sin capacidad para decidir, ni tampoco para equivocarse.

Sin embargo, dentro de este proceso de socialización y de la sociedad misma, no todos los niños y adolescentes pudieron hacerle frente de igual forma, y es aquí donde se encuentran los estudiantes con alguna excepcionalidad, porque aprenden muy rápido, o los que tienen alguna discapacidad -la mayor parte de veces creada por la sociedad- ya sea física, cognitiva o de conducta. "...sé, que debo portarme bien y lo que debo hacer... pero, no sé qué me pasa... termino haciendo lo que no debo, ... yo quiero, pero no puedo..." (conversación con Juan Carlos, 8vo. grado, colegio privado de Lima, con diagnóstico de Trastorno por Déficit de Atención con Hiperactividad (TDAH) y Trastorno Oposicionista Desafiante (TOD).

El caso de Juan Carlos, es muy similar al de muchos niños y adolescentes con diagnósticos similares o muy distintos (dislexias, problemas de procesamiento de la información, trastorno de ansiedad generalizada, depresiones, discalculia, disfluencia, psicosis, sin contar los casos de estudiantes con algún tipo de discapacidad física, etc.), para los cuales la escuela actual no siempre se encuentra preparada, y es más, muchos de ellos se encuentran obligados a tomar medicamentos varias veces al día para poder obedecer y estar tranquilos en clases en las que los estudiantes pasan entre seis a ocho horas con metodologías repetitivas y no adaptadas al grupo de estudiantes y a sus peculiares necesidades, sino al gusto del profesor, el cual, en su mayoría, sobre todo en secundaria, se centra sólo en su curso, sin trabajar de forma interdisciplinaria y conectada a través de proyectos $\mathrm{u}$ otros con otros docentes, lo que le permitiría al estudiante desarrollar el aprendizaje como acción, y no de manera pasiva.

No resultan extrañas las clases, sobre todo en el nivel secundario, con presentaciones elaboradas en Power Point, algunas preguntas para ser respondidas en fichas $\mathrm{u}$ organizadores visuales de forma grupal o dando exámenes escritos de forma individual, en columnas.

Son escasos los profesores y las instituciones educativas (tanto de educación básica como universitaria) que se atreven a realizar evaluaciones cooperativas a través de productos finales, en muy pocos casos, se usan herramientas distintas a Kahoot o Padlet, claro, siempre y cuando cuenten con proyector y computadora en el aula. Desarrollar clases fuera de las aulas, se convierte en ocasiones, en lo mismo que dentro del salón, pero en el patio o en el jardín, si es que lo tienen, es un cambio de ambiente -que ya es bueno-, pero con la misma actividad, resulta siendo "más de lo mismo".

Lo anterior tampoco libra a los padres de familia y a la escuela como tal de buena parte de la responsabilidad, ya que los primeros, en la búsqueda de dar una mejor calidad de vida a su familia, dejan de lado a aquello, que supuestamente les da el propósito, los hijos, 
permitiendo que pasen en la institución escolar, en algunos casos, hasta nueve horas diarias, por lo que, suponen, que la escuela debe realizar la función formadora que les corresponde a los padres, siendo esto un imposible, es decir, la función paterna está puesta en juego.

Por otro lado, la escuela, en caso de ser privada, sólo se adapta a lo que los padres quieren, de eso en el Perú, hemos aprendido bien la frase "el cliente tiene la razón", olvidando la función básica de la escuela, que es la de educar.

Si nos ponemos a pensar, el ideal educativo de tener aulas con estudiantes siempre tranquilos $\mathrm{y}$ atentos, con rendimiento promedio o bueno, como se quería hace más de treinta años es irreal, y por demás, exclusivo, ya que desde siempre ha existido diversidad cerebral, no hay dos cerebros iguales, por ende, ninguna persona aprende igual que otra, quizá de forma similar, más no igual, lo que significa que algunos aprenderán mejor de forma visual, otros de forma activa, otros a través de juegos o por texturas, o a través de organizadores visuales, otros escuchando, meditando, hablando, pero sin duda, todos aprenden haciendo, construyendo, proponiendo ideas desde su peculiaridad.

Además, debe agregarse que los contextos de desarrollo del estudiante tampoco son iguales, padres, ambiente físico, familia y todo lo que ello implica, todo esto sumará a las diferencias que tiene cada estudiante y a las diferencias tanto de cerebro como psíquicas, por lo que pensar en homogeneidad frente a la diversidad resulta absurdo y retrógrado.

En base a lo anteriormente dicho, cabe preguntar ¿Hasta qué punto la educación basada en la diversidad es determinante para evitar el fracaso académico de estudiantes?

\section{El imperativo de éxito en los "estándares" de la diversidad}

La realidad es una experiencia creada por nuestro cerebro. López, D. (2019). Equilibrio.
Lo diverso no tiene estándar, pero el imperativo de éxito, sí. Si no hay dos cerebros iguales, ¿por qué el concepto de éxito debería ser igual?

Quizá se puede notar que la pregunta en la sección anterior parte de una contradicción en sí misma, ¿cómo se puede hablar de fracaso escolar en una educación basada en la diversidad? Sin duda, así es, no tiene sentido, pero cobra sentido en la primera oración del párrafo anterior.

Como lo explica Ken Robinson, en el video Cambiando Paradigmas (Recuperado de: https:// www.youtube.com/watch? $\mathrm{v}=$ Z78aaeJR8no), desde la Revolución Industrial, el cuidado por la calidad se convierte en una exigencia a todo aquel que hace empresa, esto no ha cambiado mucho, quizá solo ahora se realiza de formas más sofisticadas, pero la exigencia por la calidad se ha mantenido constante, el día de hoy incluso, manejamos estándares nacionales e internacionales para poder hacerle frente a esa calidad, la cual se evalúa por procesos, atención al cliente, producto final, etc.

Esto no quiere decir que la exigencia y condiciones laborales no hayan cambiado, de eso no cabe duda, lo que lleva al desarrollo de otras habilidades por parte de cada generación que ingresa al mundo laboral industrializado y digital, habilidades que en buena medida están en manos de las escuelas y de la enseñanza superior, lo que implica otro tipo de desafío, ya que muchas de esas habilidades que pide el mercado laboral actual, son las llamadas habilidades blandas.

En educación, como se ha mencionado, no estamos al margen de la exigencia social, existen estándares desde inicial hasta posgrado, claro que no todas las instituciones pueden acceder a estas estandarizaciones debido básicamente al costo de la acreditación, su implementación y, por supuesto, su mantenimiento.

En esta línea, de medición, el estado peruano aplica, de forma obligatoria, la Evaluación Censal de Estudiantes (ECE), como una forma 
para medir los niveles de aprendizaje de los estudiantes en ciertas disciplinas, a lo largo de un año.

La forma como está planteado deja de lado lo peculiar del estudiante y lo que es el mismo proceso de aprendizaje. En ese sentido el neurólogo argentino Daniel López, afirma en su texto Equilibrio (2019), “...lo que vivimos, tanto en nuestro ámbito de orden racional o emocional, modifica nuestros circuitos..." (p. 61) y respecto al aprendizaje nos dice que "... el proceso de aprendizaje... consiste, como vimos, en una remodelación de los circuitos cerebrales..." (p. 62).

De lo anterior, se desprende que no se mide de forma adecuada lo aprendido, sino que más bien mide lo acumulado, yendo en contra del mismo estudiante y su proceso individual de aprendizaje.

Para profundizar en el punto, quizá cabe preguntar, ¿qué se viene entendiendo por aprendizaje? Una idea que podemos tomar como factor común de las distintas concepciones, es que se trata de un proceso, que tiene efectos físicos claros en el cerebro como lo deja ver López (2019), "es necesario lo racional y las emociones para lograr esa modificación de circuitos", y agrega, "esa modificación, que implica la unidad de nuestra mente y nuestro cerebro, da lugar a una red nueva de conexiones cerebrales que serán responsables de un fenómeno extraordinario: modificarán el entendimiento que hagamos sobre nuestras experiencias futuras" (p. 61).

En ese sentido, Robinson (2015) afirma: "los seres humanos somos organismos vivos con una gran curiosidad por aprender. Desde que nacen, los niños tienen una sed de aprendizaje inagotable. Para muchos de ellos, demasiados, su paso por la escuela va apangando esa sed" (p. 18), y es como también lo explica Ken Robinson, el modelo educativo, aún imperante, nos habla de estudiantes que sirven para estudiar y otros que no (pp. 19-20).
Entonces, no basta la razón y lo académico, son necesarias las emociones, y lo que lo logra es el aprecio por la vivencia, las experiencias, el aprender haciendo. En ese sentido, ¿cómo se podría evaluar lo hecho con sólo teorización?, de hecho, la teoría, lo académico es importante para sustentar el hacer, pero no basta, es una evaluación incompleta. Por lo que entender el aprendizaje como saber académico es un reduccionismo que en educación no podemos darnos el lujo de seguir manteniendo, ya que minimiza el proceso de enseñanza-aprendizaje para aquellos cuyas habilidades se encuentran en el orden del saber académico, dejando de lado a la gran mayoría que aprende de forma distinta, y que podría no ser tan efectivo en el logro de este tipo de saberes pero que pueden resultar con aprendizajes valiosos en diversos campos como el artístico, el cinestésico corporal o en algún otro, del cual, sin duda podría realizar valiosos aportes.

Desde un imperativo de calidad, al que han ayudado mucho los medios de comunicación, se nos ha hecho creer a lo largo de muchos años que sacar buenas notas, o que ingresar a la universidad entre los primeros puestos, asegura el éxito en la vida, "los que son muy inteligentes van a universidades prestigiosas... Los que se gradúan con buena nota tienen asegurado un trabajo profesional bien remunerado con despacho propio" (Robinson, 2015, p. 20).

Lo anterior difiere mucho, con alguno de los íconos actuales de éxito: Steve Jobs, Bill Gates o Mark Zuckerberg, quienes abandonaron sus estudios universitarios para dedicarse a sus empresas hasta convertirse en multimillonarios $\mathrm{y}$ en gente muy reconocida en los diferentes ámbitos donde se desenvuelven.

No obstante, ¿sólo se puede considerar exitosa aquella persona que logró lo que Jobs, Gates y Zuckerberg? Sin duda, el lector coincidirá que esto sería igual que una evaluación sólo del saber académico, por lo que hablar de éxito también variará de persona a persona, o de cerebro a cerebro, no es posible generalizarlo. 
Gastón Aramayo y Victoria Morales, se han dedicado por cincuenta años a la famosa escuela de títeres Kusi-Kusi, sin tener apoyo más allá de su propio esfuerzo, ahora cierran la escuela, por ya no poderse dedicar a ella (Recuperado de: https://larepublica.pe/cultural/2020/02/25/teatro -kusi-kusi-la-funcion-de-titeres-ha-terminadogaston-aramayo-y-victoria-morales/).

Quien se dedica por tanto tiempo a algo, es innovador -en el sentido de crear lo que no existía o era habitual previamente a su idea-, busca hacerlo bien, y es considerado un referente, ¿también puede ser considerado exitoso?

¡Qué duda cabe!, lo diverso está también presente en el éxito, no hay una única forma de ser exitoso, aunque se nos repita, sobre todo en la publicidad y a través de los medios de comunicación. Ser famosos, gastar, viajar y comprar muchas cosas, no constituyen la única forma de ser exitosos.

Hace poco, me llamó mucho la atención la entrevista que le realizaron a Ricardo Darín. Reconocido actor argentino, quien mientras trabajaba haciendo teatro en Madrid, le ofrecieron actuar para el cine en Hollywood, siendo su respuesta negativa, aunque le insistieron y le ofrecieron más dinero, él tampoco aceptó, ante tal respuesta el entrevistador no supo qué decir, al constatar que Darín apreciaba más otros aspectos de la vida, y que sin tanto dinero, y sin tanta fama, él se sentía feliz, un hombre exitoso, al poder trabajar en España y que le fuera bien, y por tener una familia que le daba sentido a su vida, "soy todo lo feliz que se puede ser y tengo mucho más de lo que necesito" “...soy un tipo privilegiado...”. (Ricardo Darín, Sobre Hollywood y el valor de la vida. Recuperado de: https://www.youtube.com/watch?v=xDVLOg6jGU).

El éxito no se mide en dólares, sino en lo que cada uno desea de forma particular. La educación en la diversidad alienta la peculiar forma de éxito de cada estudiante, no hay rótulos, sólo existen personas únicas.
"La finalidad de la educación es capacitar a los alumnos para que comprendan el mundo que les rodea y conozcan sus talentos naturales con objeto de que puedan realizarse como individuos y convertirse en ciudadanos activos y compasivos" (Robinson, 2015, p. 24).

Es decir, lo que conforma a cada estudiante se convierte en su propio estándar, sus habilidades y talentos no son más que su justa medida, en donde su éxito o fracaso solo puede ser establecido por el propio estudiante y la constatación del desarrollo de sus habilidades. Quizá, aquí se encuentre la función que tiene el Estado, la de facilitar el desarrollo de habilidades y talentos de cada persona que forma parte de la sociedad, a fin de que pueda sentirse exitosa y feliz en el país que habita, sin necesidad de sentirse excluida por tener que ajustarse a parámetros extraños al propio avance.

\section{Escuchar la escuela, escuchar lo diverso como Uno.}

\section{"Lo que es importante para el alumno tiene que serlo también para nosotros". Robinson, K. (2015, p. 32).}

La frase con la que empieza esta sección es, en especial significativa, ya que podríamos decir que es el significante del cambio que logró la directora de un colegio en el estado de Georgia en los Estados Unidos.

Robinson (2015) cuenta la experiencia de la doctora Laurie Barron, quien junto con sus profesores lograron que la escuela Smokey Road cambiara su mala reputación, bajos resultados académicos y alto ausentismo, al punto de convertirse en una escuela distinguida (pp. 33-34). ¿Cómo lograron ese cambio?, en una palabra, escuchando.

La clave para Laurie y para cualquier escuela es, Escuchar, ¿Escuchar qué? Lo más importante, al estudiante y al maestro y desoír la lógica normalizada, como dice Robinson, o la burocracia como expresa Duschatzky, que suele 
expresarse en currículos o programas poco o nada flexibles para la realidad de los estudiantes, quizá porque a ese currículo le falta escuchar al estudiante, y lograr el encuentro, convergencia en la que se da el aprendizaje.

El encuentro para el aprendizaje sólo es posible cuando se reconoce la diferencia en cada individuo, y su peculiar forma de aprender y ser, esto implica necesariamente un especial énfasis en el docente y la metodología que utilice, esa metodología que le permita al estudiante adquirir ese aprendizaje, hacerlo suyo, es una decisión, única, propia, no es algo impuesto que viene desde una autoridad, porque "debe de darse", es algo que viene desde una decisión particular, es así como lo plantea Larrauri (2001), la hablar de Giles Deleuze y su enseñanza.

Y, como Sócrates, sabía que cada cual tiene que aprender a pensar por sí mismo y que, por lo tanto, enseñar no es comunicar, ni informar, sino discurrir, dejar que el discurso discurra ante los oyentes para que sea el propio oyente el que decida en qué momento entra en la corriente del pensamiento. (p. 1)

En ese sentido, Deleuze tenía muy claro que también el docente y su metodología son muy importantes, por este motivo, Larrauri, refiriéndose a Deleuze señala que:

Sus clases estaban muy preparadas. Concebía su preparación como un ensayo continuo, como hace un actor para meterse en la cabeza lo que tiene que decir, de manera que cuando lo despliega ante el público se apasiona con lo que dice. (2001, p. 1)

Cuando Giles Deleuze inicia sus clases en la Universidad de Vicennes en París, tenía un público muy variopinto, formado por intelectuales, profesionales, obreros e incluso estudiantes de los últimos años del colegio (bachillerato), y para este público tan diverso preparaba sus clases para que todos discurrieran, y decidieran aprender, no eran clases llenas de jerga filosófica, sino basadas en la realidad, en lo cotidiano, facilitando al estudiante aprender y lograr un contacto.

Como se deja ver en el caso de Deleuze, prepararse para hacerle frente a la diversidad resulta clave para lograr un impacto en los estudiantes, el ser escuchado, respetado en la diferencia, no se convierte en temor al momento de decidir, aunque esta decisión, a veces sea un desastre; pues, como afirma Blanchot, "el desastre no es sombrío..." (Duschatzky, 2017, p. 55), entendiéndose por desastre, una mala decisión, un error, una molestia, no tendría por qué ser desastrosa, ya que permite pensar, ver lo que sucede desde una óptica distinta.

Esto último, como se verá, va a contrapelo del currículo normalizado y burocrático, que castiga el error, la molestia, dejando de lado la posibilidad de aprender de ese desastre, del mal-entendido, de una de las posibilidades más comunes que tiene el ser humano, equivocarse.

El tener siempre una respuesta precisa, adecuada a la situación, donde todo esté perfectamente controlado, deja de lado la posibilidad, no hay posibilidad al desastre, siendo esta una forma de negar la condición humana, por lo que escuchar lo diverso en educación es también un acto ético, en la medida que desarrollar el carácter (ETHOS), se realiza a través de la práctica, o sea a través de ensayo y error, única forma para llegar a ser virtuoso, y en buena medida, de eso se trata aprender, de construir, dejando siempre la posibilidad de seguir aprendiendo, es decir a través de él conocer el error y mejorarlo.

En el desastre se asoma el afuera de lo familiar, si pescamos que el descalabro no es una calamidad sino la manifestación del dislocamiento de creencias y modalidades de estar en el mundo. (Duschatzky, 2017, p. 55)

\section{CONCLUSIONES}

Como podemos apreciar, el educar en la diversidad es determinante para el fracaso escolar, es un sin sentido, ya que sólo existe 
fracaso escolar en la medida en que existe estándar que superar o normalidad que seguir, en contra, incluso, de lo que las neurociencias nos enseñan, que no existen dos cerebros iguales.

Es decir, el fracaso escolar solo puede ser pensado dentro de una lógica de competencia, en donde hay mejores y peores, dejando de lado cualquier forma de peculiaridad e individualidad de aprendizaje, a una única forma de aprender y hacer las cosas, como lo dice un currículo que, muchas veces se encuentra desconectado de las necesidades reales de los estudiantes, salvo la presencia de algunas metodologías alternativas y algunas teorías que se encuentran conectadas con la nueva lógica de la normalidad.

Como consecuencia de una antigua lógica de la normalidad, se sigue pidiendo a los estudiantes buenas notas a través de evaluaciones que valoran el resultado final, más que el proceso,

\section{REFERENCIAS}

Darín, R. Sobre Hollywood y el valor de la vida. Recuperado de: https://www.youtube.com/ watch? $\mathrm{v}=\mathrm{xDVLOg} 6-\mathrm{jGU}$

Duschatzky, S. (2017). Política de la escucha en la escuela. Buenos Aires, Argentina: Editorial Paidós.

Educación (2019-1). Enseñanza-aprendizaje para la diversidad. Un pendiente educativo en la educación peruana. Lima, Perú.

Larrauri, M. (2001). El deseo según Giles Deleuze. Recuperado de: https:// alinkisforever.files.wordpress.com/2010/07/ deseo-larrauri-deleuze.pdf

López, D. (2019). Equilibrio. Buenos Aires, Argentina: Editorial Planeta.

Morales, V. Kusi Kusi, la función de titeres ha terminado. Recuperado de: https:// y más que la creación de un producto que sea la expresión real de lo aprendido, además se corta la creatividad y la acción de crear, que no son lo mismo, a fórmulas enseñadas por un profesor desde su propia subjetividad, "la creación es inmanente al problema, la creatividad es un recurso para sobrevivir" (Duschatzky, 2017, p. 39). En la educación burocrática, tanto la creación como la creatividad son cortadas.

Hablar de la diversidad, es hablar de lo peculiar que debe escuchar la escuela, la posibilidad de decidir aprender, de diversas formas, posibilidad que la escuela debe soltar a los estudiantes como forma de construir aprendizajes desde lo diverso, en donde no hay materias más importantes, en donde el mundo se construye "haciendo" de forma multi e interdisciplinaria, en donde aprender es un acto no de sobrevivir, sino de vivir, de desear profundamente.

larepublica.pe/cultural/2020/02/25/ teatro-kusi-kusi-la-funcion-de-titeres-haterminado-gaston-aramayo-y-victoriamorales/

Robinson, K., Aronica, L., \& Pérez, R. P. (2015). Escuelas creativas: La revolución que está transformando la educación. Barcelona, España: Grijalbo.

Robinson, K. Cambiando Paradigmas. Recuperado de: https://www.youtube.com/ watch? $v=$ Z78aaeJR8no

Temas para la educación (2009). Aprendizaje: Definición, factores y clases. Recuperado de: https:/www.feandalucia.ccoo.es/docu/ p5sd4922.pdf

Fecha de presentación: 19-02-20

Fecha de aceptación: 11-05-20 